\title{
Equine strangles: An update on disease control and prevention
}

\author{
Maria C. Duran ${ }^{a^{*}}$, Lutz S. Goehring ${ }^{\mathrm{b}}$
}

\begin{abstract}
Streptococcus equi spp. equi (SEE) causes a disease in horses commonly referred to as strangles. Carrier or reservoir equids are important for the maintenance of the bacteria between epizootics and the initiation of outbreaks on premises, they also make the control and prevention of the disease more difficult. Disease outbreaks are common in many countries, affecting negatively equine health and causing major economic losses to the equine industry. This review describes general aspects of the disease caused by SEE in horses (clinical signs, pathogenesis, epidemiology, treatment, complications) and then focuses on prevention, control and eradication mechanisms.

Key words: Streptococcus equi spp. equi, lymphadenopathy, guttural pouch, empyema, strangles.
\end{abstract}

\section{GENERAL BACKGROUND}

Over the years our knowledge and understanding of equine infectious diseases have been increasing, but despite these advances, the control of many important equine infectious diseases remains challenging. Furthermore, a number of significant infectious diseases are (re-)emerging in many countries and regions, causing detrimental effects on animals' welfare and impacting negatively the equine industry.

The nature of the equine industry, where horses are regularly moved to and from competitions or between breeding farms, makes it difficult to control and prevent highly contagious infectious diseases. Incidents of disease introduction associated with national and international movement of horses are regularly reported ${ }^{1}$ (Dominguez et al 2016, Moreira et al 2019, Pusterla et al 2011, Christmann and Pink 2017). Nevertheless, the spread of infectious and contagious diseases carried by animals with subclinical infection remains a challenge for national and international trade, in part because government prevention and control programs are mainly focused on exotic diseases, whereas the control and prevention of endemic diseases (e.g. equine influenza, equid herpesvirus-infection, contagious equine metritis, strangles) still needs more attention.

Streptococcus equi spp. equi (SEE) causes the disease commonly referred to as strangles (also paperas, gurma, gourme, Druse, etc.), which is a highly infectious and contagious bacterial infection that affects horses, donkeys

Received: 14.08 .2020$.

Accepted: 02.12.2020.

${ }^{a} E q u i n e$ Clinic, Veterinary Teaching Hospital, Clinical Sciences Institute, Universidad Austral de Chile, Valdivia, Chile.

${ }^{b}$ Equine Clinic, Ludwig-Maximilians-University, Munich, Germany. *Corresponding author: MC Duran; Fundo Teja Norte s/n, Valdivia, Chile; carolina.duran@uach.cl

1 Animal Health Trust. 2019. Equine influenza outbreaks reported in 2019. Available at: https://www.aht.org.uk/wp-content/uploads/2019/04/ Equiflunet-outbreaks-to-15-April-2019.pdf and mules of any age. The disease has a worldwide distribution but outbreaks may occur when large numbers of horses are gathered together or re-introduction after population immunity has decreased (Dominguez et al 2016, Pusterla et al 2011, Boyle et al 2018). The bacterium is highly contagious and produces high morbidity and low mortality in susceptible populations previously free of disease. Transmission occurs via direct contact with infectious exudates and via fomite transmission. After infection, some animals keep harbouring the bacterium in their upper airways (more commonly in their guttural pouches). Carrier animals are important for maintenance of the bacteria between epizootics and initiation of outbreaks on premises, they also make the control and prevention of the disease more difficult (Boyle et al 2018, Ivens et al 2011, Boyle et al 2009). The disease causes major economic losses to the equine industry worldwide due to its prolonged course, extended recovery period and associated serious complications.

The review describes general aspects of the disease caused by SEE in horses (clinical signs, pathogenesis, epidemiology, treatment, complications) and then focuses on prevention, control and eradication mechanisms.

\section{CLINICAL SIGNS}

Infection with SEE can occur in horses of all ages, more severe clinical signs are seen commonly in younger horses (Sweeney et al 2005, Pusterla et al 2011) whereas older horses are often less severely affected and recover more rapidly, probably due to their immune status (Pusterla et al 2011, Boyle et al 2018).

Clinical signs vary among animals but are generally characterised by abrupt onset of pyrexia followed by pharyngitis and abscess formation in the mandibular and retropharyngeal lymph nodes (Boyle et al 2018, Sweeney et al 2005). The first signs, 3 to 14 days after exposure, are lethargy and fever $\left(>40^{\circ} \mathrm{C}\right)$ (Waller 2014). Pharyngitis and lymphadenopathy develop in most horses, causing reluctance to eat, drink, abnormal position of the head (neck extension) and even upper airway obstruction (strangulation 
- strangles) necessitating a tracheostomy in severe cases (Mallicote 2015). Nasal discharge is common, only some horses develop cough (Mallicote 2015, Sweeney et al 2005, Boyle et al 2018). Inflammation of the larynx and pharynx makes this area sensitive to the touch, and palpation usually causes pain, stridor, gaging and cough. Although lymphadenopathy commonly develops in mandibular and retropharyngeal lymph nodes, parotid and cranial cervical lymph nodes can also be affected. Abscesses have a firm capsule and generally rupture 1 to 4 weeks after infection into the airway/guttural pouch (causing thick nasal discharge) or break through the skin (mandibular or parotid lymph nodes) (Judy and Chaffin 1999).

Complications like neuropraxia (laryngeal hemiplegia, dysphagia, or both) and damage to the recurrent laryngeal nerve with subsequent paralysis of the arytenoid cartilage may occur, contributing to breathing difficulty (Judy and Chaffin 1999, Mallicote 2015). Infection with SEE can spread occasionally to other locations, abscesses can form in multiple locations (abdomen, mammary gland, brain, etc.), a condition that is commonly called "bastard strangles". Also, SEE pneumonias and immune-mediated vasculitis leading to limb or head swelling have been described (Sweeney et al 2005).

\section{PATHOPHYSIOLOGY AND IMMUNITY}

SEE, a $\beta$-hemolytic Lancefield group C Streptococcus, is generally associated with disease in equids and is not considered a normal commensal of the respiratory tract. Its hyaluronic capsule and surface protein SeM enables SEE to evade phagocytosis, while many other cell surface antigens contribute to its virulence. Intensive research has been directed toward identifying cell surface antigens for developing better diagnostics and vaccines.

The bacteria enters the nose or mouth, then it attaches to and invades the cells within the tonsillar crypts of the lingual and palatine tonsils and the follicular epithelium of the pharyngeal and tubal tonsils, reaching the lymph nodes of the head and neck within 3 hours after infection (Timoney and Kumar 2008). There is no colonisation before penetration, thus a few hours after infection SEE is difficult to detect on the mucosal surface via culture but potentially visible within epithelial cells and subepithelial tonsillar follicles (Timoney and Kumar 2008). Hence, nasal or nasopharyngeal samples may be culture negative in the early stages of infection (Timoney and Kumar 2008, Boyle et al 2018). A few hours later, SEE is then translocated to the mandibular and retropharyngeal lymph nodes that drain the pharyngeal and tonsillar region. The interaction of complement with SEE causes migration of large numbers of polymorphonuclear neutrophils, causing abscessation, visible 3 to 5 days after SEE has entered the lymph nodes (Boyle et al 2018, Sweeney et al 2005).

SEE releases a hyaluronic acid capsule, antiphagocytic SeM protein, $\mathrm{H}$ factor binding Se18.9, Mac protein, and other undetermined antiphagocytic factors that modulate the proliferation and activity of neutrophils and macrophages (Timoney and Kumar 2008, Waller 2014), causing failure to phagocytose and kill the bacteria (Waller 2014, Boyle et al 2018). Relatively few organisms are present at the time of initial colonisation, but substantial bacterial propagation by the time of onset of fever has been detected (Mallicote 2015).

With lysis of the abscess capsule and evacuation of the contents, bacteria are eliminated. Nasal shedding of SEE begins 2-3 days after onset of fever and persists for 2-3 weeks in most animals. Shedding may persist much longer should infection persist in the guttural pouch or a sinus cavity (Chanter et al 1998, Newton et al 1997). Some animals with preexisting immunity and without clinical signs never exhibit detectable shedding (Boyle et al 2018).

Rupture of the abscesses allows for easy contamination of the environment and infection of other horses (Boyle et al 2018, Mallicote 2015).

Most horses recover from strangles over a period of weeks $(\sim 98 \%)$. Two to 3 weeks after infection mucosal and systemic immune responses are detected, coinciding with mucosal clearance of SEE (Galan and Timoney 1985, Boyle et al 2009). Long-term immunity to strangles is built in most horses after infection (approx. 75\%), if not treated with antibiotics (Boyle et al 2018, et al 2002, Tiwari et al 2007, Galan and Timoney 1985). Just after the convalescent phase horses are resistant to re-infection (Boyle et al 2018, Galan and Timoney 1985).

Despite the development of antibody responses, some horses fail to clear all abscess material from their guttural pouches or sinuses (approx. 10\%) and the residual purulent material forms chondroids that can remain in the horse for several years and even a lifetime (Newton et al 1997, Verheyen et al 2000). SEE persists in chondroids (visible) or in mucosal surface biofilms (invisible - inapparent) and is intermittently shed from carriers into the environment. Ongoing exposure to SEE due to the presence of carriers likely contributes to the maintenance of increased levels of immunity and extended strangles-free status within isolated herds of previously infected horses. Older horses, with waning immunity, and vaccinated animals have limited susceptibility to SEE and can develop a mild form of strangles ("atypical or catarrhal strangles"). Nevertheless, these animals still shed virulent SEE, able to cause severe disease in more susceptible horses (Sheoran et al 1997).

Recovered mares shed immunoglobulin $\mathrm{Gb}(\mathrm{IgGb})$ and IgA in their milk and colostrum, with specificities similar to those found in nasopharyngeal mucus of convalescent horses (Galan and Timoney 1987). Milk and colostral antibodies protect suckling foals during the first weeks and months of their lives (Boyle et al 2009).

SEE does not survives for long in the environment (surfaces exposed to direct sunlight), but it can persist for up to 1 month in sufficiently moist areas (Weese et al 2009). Transmission of SEE to naïve horses from acutely 
or persistently infected ones is via direct contact (nose or mouth), or through contaminated drinking water, tack, and other fomites.

The persistent SEE infection status is critical to interepizootic transmission, the recurrence and the high incidence of disease, making it very challenging to control and prevent the disease around the world.

\section{COMPLICATIONS}

Most strangles cases progress as described above and resolve after rupture of the abscessed lymph nodes (Boyle et al 2018, Mallicote 2015, Waller 2014). However, complication rates vary with the duration and intensity of exposure to SEE, increasing up to $20 \%$ in some cases (Sweeney et al 1987, Piche 1984, Ivens et al 2011). Case fatality rates, although generally low, can be as high as $10 \%$ during farm outbreaks (Boyle et al 2018, Christmann and Pink 2017). Isolation of infectious horses is therefore critical in reducing the complication and case fatality rates (Boyle et al 2018).

After SEE infection various sequelae can occur: establishment of chronic carriers, spread of infection from the head and neck region to other locations (metastatic abscessation), and immune-mediated complications.

\section{METASTATIC ABSCESSATION}

Commonly referred to as "bastard strangles", it occurs when the infection spreads to lymph nodes or tissues distant from the lymph nodes of the head and neck. Diagnosis is reached with a history of exposure to SEE and laboratory results consistent with chronic infection, anaemia, fever that responds to penicillin, hyperfibrinogenemia, and hyperglobulinemia. Treatment requires long-term antimicrobial therapy, and appropriate local treatment or drainage of abscesses if possible. Nevertheless, bastard strangles often results in the death of patients, especially when lungs, lymphoid tissue including the spleen in the abdomen, liver, kidneys, or brain are involved (Waller 2014).

\section{IMMUNE-MEDIATED COMPLICATIONS}

Purpura haemorrhagica (aseptic necrotizing vasculitis). It is the most frequent type of immunologic complication (immune-mediated type III hypersensitivity reaction) in response to several different SEE antigens, including anti-SeM antibody responses that result in deposition of immune complexes in blood vessels causing severe vasculitis, substantial ventral oedema, and necrosis (Sweeney et al 1987, Mallicote 2015, Pusterla et al 2003). It occurs most frequently 3 to 4 weeks after strangles or the administration of strangles vaccine (Mallicote 2015). Animals hypersensitised to SEE antigens (titers greater than $1: 1,600)$ are at increased risk of developing purpura haemorrhagica (Sweeney et al 2005). Treatment includes corticosteroids (dexamethasone) and supportive care (intravenous fluids, hydrotherapy, bandaging, etc.). Most horses can recover if given good veterinary care, but mortalities have been reported (8-25\%) (Waller 2014, Pusterla et al 2003, Sweeney et al 1987, Heath et al 1991).

Myositis. Muscle infarctions, rhabdomyolysis with acute myonecrosis, and rhabdomyolysis with progressive atrophy after SEE infection are a relatively rare, localised immunologic complication that presents as various syndromes (Mallicote 2015). Although infarctions are a severe manifestation of purpura hemorrhagica, the mechanisms of rhabdomyolysis are not known, but inflammatory cascades as with streptococcal toxic shock syndrome or direct toxic effects of SEE in muscle tissue have been hypothesised (Boyle et al 2018). Widespread lymphocytic inflammatory infiltrates with marked change most evident in atrophied muscle samples have been reported in the histopathologic evaluation of muscle tissue (Mallicote 2015). Horses with myositis should be treated with corticosteroids, if there are signs consistent with concurrent infection, antibiotics are also indicated (Boyle et al 2018).

Myocarditis. Antigens of SEE were also reported to trigger inflammation of the myocardium, causing electrocardiographic abnormalities in convalescent horses (Boyle et al 2018, Mallicote 2015).

\section{DIAGNOSIS}

The diagnosis of SEE infection traditionally relied on bacterial culture out of material recovered from swabs, washes from the upper respiratory tract or abscess content. Advances in molecular technology (quantitative PCR or other PCR formats) have shown deficiencies in the culture test, proving that this method is no longer the gold-standard method for the detection of SEE, diagnosis of strangles or to detect carrier status. Serology is used to identify exposure, higher titers have been found in carrier animals and are also associated with a higher risk of developing immunomodulated complications. While still cited by some as the gold standard of diagnosis, culture results must be carefully interpreted, especially when used to screen samples collected from the upper respiratory tract.

\section{CULTURE}

Culture details are reported elsewhere (Mallicote 2015, Boyle et al 2018, Waller 2014). Briefly, samples are cultured in blood agar containing colistin and nalidixic acid. After overnight incubation, beta-haemolytic colonies of SEE are picked and used to inoculate Todd-Hewitt nutrient broth. Turbid cultures are also used to inoculate purple broth cultures containing trehalose, lactose, or sorbitol (SEE fails to ferment these sugars, whereas $S$. zooepidemicus ferments lactose and sorbitol, and $S$. dysgalactiae subsp. S equisimilis ferments trehalose) (Bannister et al 1985). 
The isolation and identification of SEE is time consuming (at least 48h) and confounded by the presence of other beta-hemolytic bacteria ( $S$ zooepidemicus, $S$ equisimilis) that tend to overgrow SEE in culture. In samples collected from the upper respiratory tract SEE has to outcompete other normal respiratory flora in order to be readily identified in culture. Thus, culture results must be carefully interpreted, especially when used to screen samples collected from the upper respiratory tract (Boyle et al 2018, Mallicote, 2015). The reporting delay could also have consequences for the spread of SEE if suspected animals are not isolated when samples are collected.

\section{PCR ASSAYS}

Different PCR-based tests have been developed. The first test that targeted the 50 region of the SeM gene was reported to be 3 times more sensitive than culture (Båverud et al 2007, Webb et al 2013). However, the region is highly variable, and some SEE strains even lack the target. Therefore, a variety of other sequences and qPCR formats were developed to assure even greater specificity and sensitivity (Webb et al 2013, Båverud et al 2007), also providing fast results (qPCR test can be completed within 1-2 hours, results may be available on the same day that samples arrive at the laboratory) (Boyle et al 2018, Webb et al 2013, Mallicote 2015, Waller 2014).

Recently, a triplex qPCR assay was developed (Webb 2013) that targets two SEE specific genes (eqbE, SEQ2190) and an internal control strain of S. zooepidemicus (within-assay control, to reduce false-negatives). This assay has an overall sensitivity and specificity of 93.9 and $96.9 \%$, respectively, and is able to detect 10-fold fewer quantities of SEE than culture, regardless of the presence of contaminating bacteria (Webb et al 2013). Therefore, the triplex qPCR sets a new benchmark for quality control and sensitivity and is now regarded as the new gold-standard test for the detection of SEE (Waller 2014). Culture assays failed to identify $39.7 \%$ of qPCR-positive samples (Webb et al 2013). Historically, this poor sensitivity of culture assays and its failure to correctly identify qPCR-positive samples was excused by the claim that PCR does not distinguish between dead and live organisms, and so technically false-positive reactions affect the diagnostic value of PCRs with respect to detection of actual infection (Sweeney et al 2005). Although this is technically correct, as DNA does not persist on mucosal surfaces, experts emphasise that any culture-positive or qPCR-positive result should be taken seriously (Boyle et al 2018). Positive results of the clinical application of qPCR in the diagnosis and control of field outbreaks of strangles in several countries and management settings are considered a testament to the usefulness of qPCR over culture (Boyle et al 2018). Experts have also recommended the use of PCR testing of an endoscopically guided guttural pouch lavage for detection of SEE in subclinical infected carrier animals.
Also, the visual detection of inflammation of the guttural pouch respiratory epithelium, as well as the presence of empyema, chondroids, or enlarged retropharyngeal lymph nodes on the floor of the guttural pouch, may suggest strangles even when the lavage is negative for SEE.

\section{SEROLOGY}

Indirect enzyme-linked immunosorbent assays (iELISA) are available to detect anti-SEE antibodies (anti-SeM, anti-antigen A (SEQ2190 N-Terminal Fragment) and -antigen B or C (SeM N-Terminal Fragment).

SeM-based-iELISAs were developed to detect antibody titers to SeM that peak about 5 weeks after exposure and remain high for at least 6 months (Timoney et al 2007, Galan and Timoney 1985, Galan and Timoney 1987).

The SeM antibody titer can be used to: 1) detect recent infection (4-fold or greater increase in titers, 10-14 days apart); 2) support the diagnosis of SEE-associated purpura hemorrhagica or metastatic abscessation (titer $\geq$ $1: 12,800)$; and 3 ) identify high risk of developing purpura hemorrhagica (animals with titers $>1: 3,200$ should not be vaccinated) (Boyle et al 2018, Boyle et al 2009).

Nevertheless, false positive results were associated with cross-reactivities between anti-SeM and anti-SzM40 antibodies, produced in response to the homologue protein to SeM expressed by Streptococcus zooepidemicus (Waller 2014). This was overcome with preincubation of the serum with heat-killed $S$. zooepidemicus which allowed to remove cross-reactive antibodies from the samples (Davidson 2008), but the step was not incorporated in full-length SEM assays (Waller 2014). Therefore, a SeM-specific titer should not be used to determine carrier status and a single value cannot be used as a measure of active infection or protection to reinfection (Boyle et al 2018). SeM titers wane over time (Boyle et al 2017, Sheoran et al 1997) and horses treated with antibiotics during an outbreak seem to mount a reduced immune response, remaining susceptible to reinfection (Piche 1984).

Dual antigen or combined antigen iELISAs were then developed to overcome the cross-reactivities with $S$. zooepidemicus. This combined iELISA test detect antibodies against antigen A (SEQ2190 N-Terminal Fragment; also known as Se75.3), antigen B or C (SeM N-Terminal Fragment) of SEE, if one or both ( $\mathrm{A}$ and $\mathrm{B}$ or $\mathrm{C}$ ) antibody results exceed the cutoff, the sample is considered positive (Robinson et al 2013, Knowles et al 2010). These tests were successfully used to determine the prevalence of exposure to SEE in horse populations in Lesotho (Ling et al 2011), UK (Knowles et al 2010, Waller 2014, Ivens et al 2011) and Sweden (Riihimaki et al 2017). The antigen $\mathrm{A}$ and $\mathrm{C}$ dual antigen iELISA test was compared to a commercial iELISA (IDvet) based on the full SeM protein, showing a similar sensitivity (89.9 vs $93.3 \%$ ), but higher specificity (77.0 vs $99.3 \%$ ), highlighting the application of this dual antigen anti-A and -C antigen test to identify 
potential carriers before they can transmit the infection (Robinson et al 2013). Recently, this combined antigen $\mathrm{A}$ and $\mathrm{C}$ iELISA test was recommended to identify recent infection as early as 2 weeks after infection and exposed carrier animals without signs, provided currently available vaccines have not been used (Boyle et al 2018).

\section{TREATMENT OF HORSES WITH STRANGLES}

In animals with lymph node abscessation hot packing and topical softening agents can help in the development and maturation of the masses, speeding up the resolution (Sweeney et al 2005, Mallicote et al 2015). Surgical intervention of sufficiently mature and soft masses can be required when abscesses do not rupture spontaneously (Sweeney et al 2005, Boyle et al 2018). Once drainage occurs, daily lavage of the abscess with dilute povidone iodine solution should be instituted (Sweeney et al 2005).

The use of antibiotics in the treatment of SEE infected horses is still controversial. The bacterium is sensitive to many antibiotics, but reaching SEE in organised abscesses remains challenging. Penicillin is considered the drug of choice and antibiotic resistance has not yet been reported in SEE (Boyle et al 2018) but has begun to emerge in some strains of S. equi spp. zooepidemicus (Chalker et al 2012).

The treatment of isolated clinically healthy in-contact animals for 3 to 5 days can prevent the development of clinical signs, but it also prevents treated animals to develop SEE immunity (Sweeney 2005) and it delays abscess maturation, extending recovery times (Waller 2014, Boyle et al 2018). With severe cases, antibiotic treatment is often needed to reach clinical improvement, reducing fever, lethargy and upper airway obstruction (Mallicote 2015).

\section{PREVENTION, CONTROL AND ERADICATION MECHANISMS}

In many countries strangles is an endemic disease that generally comes in waves or outbreaks when naïve population is exposed to carrier animals. Historically, SEE was considered by veterinarians and the equine industry as a common pathogen that caused a limiting disease in young horses. Nevertheless, nowadays probably due to the increase in horse movements, waning immunity and other factors, strangles outbreaks are more common and frequent, reemerging even in countries where strangles have long been a rarity. The disease continues to cause major economic losses to the equine industry worldwide, by the direct effects of the disease (isolation, treatments, prolonged disease course, extended recovery period, serious complications) and indirectly by limiting horse movements and causing the cancellation of equestrian events.

Therefore, the authors believe national and international efforts should be directed to prevent and control the disease, starting by making the disease reportable and focusing on identifying and treating carrier animals and screening regularly possible exposure of moving equids. Shedding of the SEE from carrier animals enables the onward transmission of SEE and further outbreaks of disease. Identification and treatment of persistently infected carriers is critical to break the cycle of infection and eradicate SEE.

\section{CONTROL DURING AND AFTER OUTBREAKS}

Early identification of the infectious agent is essential to minimise the impact of the disease. Measuring rectal temperatures once or ideally twice daily aids in early identification of suspect outbreak cases.

Initial clinical signs of strangles (pyrexia, nasal discharge, and enlarged mandibular lymph nodes) vary among horses and are obviously not restricted to SEE infection. However, if infection is suspected, affected animals should be isolated and biosafety measures must be applied to minimise the transmission to in-contact animals. Confirmation of clinical cases can be achieved quickly by qPCR analysis of needle aspirates from enlarged or abscessed lymph nodes (Sweeney et al 2005). SEE rapidly invades (draining) lymph nodes of convalescent animals, hence in initial stages of disease negative results from qPCR analysis of nasal/ retropharyngeal swabs or lavages can occur, and do not necessarily rule out SEE infection, especially if clinical signs suggest otherwise (Waller 2014, Boyle et al 2018).

General biosecurity measures are described in detail elsewhere (Waller 2014, Boyle et al 2018, Mallicote 2015). Nose to nose contact and contact with nasal discharge and purulent material from erupted abscesses must be avoided.

To minimise the spread of disease, horse movements should be stopped, and farms or equine facilities can be divided into 3 color-coded groups. The red group includes horses that have shown 1 or more clinical signs consistent with strangles. Yellow/Orange/Amber group horses are those that have had direct or indirect contact with an infected horse in the red group and may be incubating the infection. Horses in the green group have had no contact with infected or suspect animals. Body temperatures of horses in the yellow and green group should be measured twice daily to identify new cases and move them to the red group. Equipment used with horses can be colour coded as well to avoid cross contamination. Ideally, different equipment and personnel should be assigned to the different groups, if not possible, staff should always move from the lowest to the highest risk group (from green to red).

Screening by dual antigen ELISA A and C iELISA to identify SEE-persistently-infected-horses can commence at least three weeks after the resolution of the last clinical case. Horses in the yellow and green groups must be included in the screening, otherwise carrier horses, which remained subclinical or were exposed before and did not get infected, will remain undetected and stay as a source of future re-infections. All horses from the red group and those from the yellow and green group that tested positive by 
iELISA should be examined further. Ideally, upper airway with guttural pouch endoscopy should be performed, followed by the collection of bilateral guttural pouch washes (Waller 2014). If not possible, nasopharyngeal swabbing/ washing samples should be collected (Boyle et al 2018, Waller 2014). Samples should be analysed by qPCR to maximise sensitivity. Identified carriers should be treated as described in figure 1.

\section{PREVENTION - ERADICATION}

Identification and treatment of carrier animals. Horses that are SEE carriers are persistently infected but seem clinically normal and generally remain unaffected during strangles outbreaks. In most farms, once clinical signs of affected animals resolve, the screening of unaffected animals is considered unnecessary but it should not be the case, as it is increasing the chances of keeping possible carrier animals and a future infection source in the population. Nevertheless, screening by dual antigen A and C iELISA has been successful in identifying persistently infected horses (Boyle et al 2009, Riihimaki et al 2017, Robinson et al 2013).

Serology for identification of infected animals. To prevent the entry of carrier animals into a herd, before entry horses should stay in quarantine and serology of blood samples collected on arrival should be performed (Waller 2014). If negative, the analysis should be repeated 14 days later to identify horses that have been incubating the infection and seroconvert after arrival. If the second sample is still negative, it should be safe to enter the new arrivals into the herd. If positive, on day zero or 14 , further diagnostics are required (Waller 2014). Endoscopy of the upper airway and guttural pouches should be examined to identify obvious signs of persistent infection (empyema, chondroids, follicular hyperplasia, etc.). During this procedure, (saline wash) samples from both guttural pouches should be taken for qPCR analysis. If visually the airways are normal and qPCR analysis results are negative, it should be safe to introduce the horse into the herd. If qPCR samples test positive or chondroids are visible on endoscopy, the horse should be treated to eliminate the carrier status before entry into the premises (Waller 2014).

Treatment of carrier animals. Once carrier animals have been identified, elimination of SEE from the guttural pouches should be accomplished by endoscopic guttural pouch lavage. If large numbers of chondroids are present, surgical hyovertebrotomy and ventral drainage through the Viborg triangle can be performed, but the procedure carries inherent risks of general anesthesia and surgical dissection around major blood vessels and nerves, plus the contamination with SEE of the hospital environment (Waller 2014).

For guttural pouch lavages, horses are sedated to facilitate drainage of flush material, if chondroids are present, they can be removed with basket tools passed through the biopsy channel of the endoscope (Waller 2014, Verheyen e et al 2000). Lavages with isotonic saline or polyionic fluid using rigid or indwelling catheters or with a suction pump attached to the endoscope should be repeated until negative qPCR results are obtained.

Topical instillation of acetylcysteine solution (20\%, weight/volume) into guttural pouches can be performed to assist empyema treatment. Once the lavage is finished,

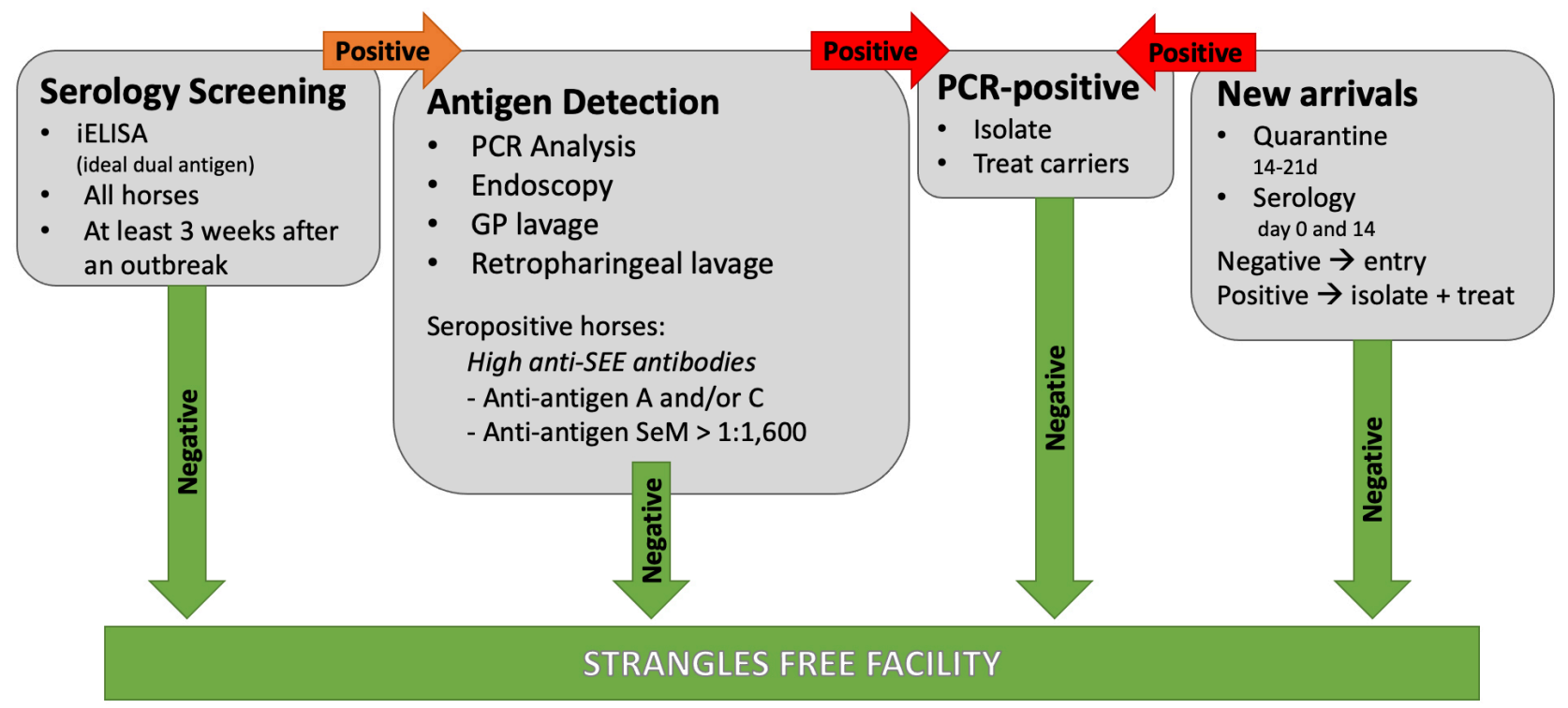

Figure 1. Diagram of procedures to reach a strangles free facility. SEE: Streptococcus equi subspecies equi; GP: guttural pouch; iELISA: indirect enzyme-linked immunosorbent assays; PCR: Polymerase C Reaction; d: days. 
topical benzylpenicillin mixture can be instilled into the guttural pouches to facilitate SEE elimination Waller 2014, Boyle et al 2018). The administration of systemic antibiotics may further improve treatment success (Waller 2014, Boyle et al 2018).

In two-weeks intervals the guttural pouches should be resampled for qPCR analysis to confirm SEE elimination. Two to 3 qPCR negative results, two weeks apart, are considered sufficient to confirm elimination of carrier status.

Vaccination. Ideally, vaccines should provide adequate levels of protection against circulating SEE strains with a long duration of immunity, especially for horses that travel and are exposed to other horses from different origins. They should also be safe to be administered and capable to differentiate infected animals from vaccinated ones, which enables normal movement of vaccinated horses but also permits the identification of vaccinated horses that were exposed to and are protected from SEE (differentiate infected from vaccinated animals - DIVA) (Waller 2014).

-Killed and cell extract vaccines: These are the first types of vaccines that were developed. Nowadays, commercially available ones which are administered by the intramuscular route, include Equivac S (Zoetis New Zealand), Strepguard (MSD Animal Health), and Strepvax II (Boehringer Ingelheim). Little is known about the real efficacy of these vaccines. However, they were reported to reduce the severity and frequency of strangles but adverse reactions were common, the protection conferred was short-lived and DIVA is not possible (Hoffman et al 1991).

-Live-attenuated vaccines: There is one live-attenuated aroA deletion mutant available in Europe based on an isolate from Holland (Equilis StrepE, Europe; MSD Animal Health) (Kelly et al 2006, Jacobs et al 2000). Submucosal and intramuscular (IM) injection protects from lymph node abscessation (Jacobs et al 2000), but adverse reactions from IM injections made this administration route less practicable (Kelly et al 2006, Jacobs et al 2000, Kemp-Symonds et al 2007). The vaccine contains the same genetic material as virulent strains of SEE, and therefore it has no DIVA properties.

Another live-attenuated vaccine is the Pinnacle IN (Zoetis), available in North America and some other countries. It is for intranasal administration, based on the CF32 strain isolated in the USA (New York 1981) and attenuated with nitrosoguanidine. Lymph node abscess formation and bacterial shedding after vaccination were reported, it caused severe adverse effects when injected intramuscularly and it does not permits DIVA (Waller 2014). Pinnacle IN vaccine-like SEE strains were isolated in strangles cases in New Zealand, suggesting an increased sensitivity to vaccine reactions and possible revert to virulence (Patty and Cursons 2014). Efficacy has not been reported, but Zoetis claims that following experimental challenge live-attenuated vaccines for strangles can confer significant levels of protection. However, they also lack DIVA properties and adverse reactions occur (Waller 2014).

Sequence analysis of the SeM gene identified differences between strains of SEE (Parkinson et al 2011, Anzai et al 2005, Kelly et al 2006, Patty and Cursons 2014, Ivens et al 2011), suggesting that the population of SEE is changing over time as the organism is evolving with dominations of SeM-9 strains in the UK (Parkinson et al 2011, Ivens et al 2011). The vaccines Pinnacle and Equilis were derived from strains that are distantly related to dominant SeM-9 strains. It is likely that antibody responses cross-react among SEE strains (Galan and Timoney 1988) but the level of protection conferred by the vaccines against circulating SEE remains unknown (Waller 2014).

-Subunit vaccines: Recombinant SEE proteins have also been produced to generate vaccines. They are safer as they only contain target proteins and no SEE DNA, making it possible to DIVA.

Nevertheless, vaccination of horses with three different subunit vaccines (recombinant SeM; SEE-specific proteins; SEE adhesin proteins) did not protect against in vivo challenge with SEE (Sheoran et al 2002, Timoney et al 2007), despite the generation of serum antibody response. The inclusion of immunoglobulin-cleaving proteins IdeE and IdeE2 in subunit vaccines were found to be important for effective protection (Guss et al 2009). A developed vaccine (known as Strangvac), based on a SeM-9 SEE strain isolated in Sweden (Guss et al 2009), included immunoglobulin-cleaving proteins IdeE and IdeE2 but not SeM or SEQ2190 proteins, to reach DIVA properties. The strain used is more closely related to the circulating strains in Europe (UK) and was found to provide protection against in vivo challenge (Guss et al 2009).

\section{CONCLUSIONS}

Strangles outbreaks are common in many countries and they continue to affect equine health negatively and cause major economic losses to the equine industry worldwide.

National and international efforts should be directed to establish effective and on-going disease surveillance together with the identification and treatment of persistently infected carriers.

Developed diagnostic tests (qPCR and serology) have greatly improved over the years and should become available and accessible worldwide to assist the identification of persistently infected equids to prevent future outbreaks.

Although strangles vaccination is used in many equine facilities to reach herd immunity, conferred protection by available vaccines is limited and their antibody response cannot be differentiated by existing serology tests. Further research is needed to generate and test effective vaccines and to use diagnostic testing alongside vaccinations that provide adequate herd immunity. 
For now, we believe that the surveillance of outbreaks as well as the testing and treating of SEE carriers can reach disease control and prevention and will lead to the break of the cycle of infection, and, eventually, to disease eradication.

\section{REFERENCES}

Anzai T, Kuwamoto Y, Wada R, Sugita S, Kakuda T, et al. 2005. Variation in the N-terminal region of an M-like protein of Streptococcus equi and evaluation of its potential as a tool in epidemiologic studies. Am J Vet Res 66, 2167-2171.

Artiushin SC, Timoney JF, Sheoran AS, Muthupalani SK. 2002. Characterization and immunogenicity of pyrogenic mitogens SePE-H and SePE-I of Streptococcus equi. Microb Pathog 32, 71-85.

Bannister MF, Benson CE, Sweeney CR. 1985. Rapid species identification of group $\mathrm{C}$ streptococci isolated from horses. J Clin Microbiol $21,524-526$.

Båverud V, Johansson SK, Aspan A. 2007. Real-time PCR for detection and differentiation of Streptococcus equi subsp. equi and Streptococcus equi subsp. zooepidemicus. Vet Microbiol 124, 219-229.

Boyle A, Sweeney C, Kristula M, Boston R, Smith G. 2009. Factors associated with likelihood of horses having a high serum Streptococcus equi SeM-specific antibody titer. J Am Vet Med Assoc 235, 73-977.

Boyle AG, Smith MA, Boston RC, Stefanovski D. 2017. A case-control study developing a model for predicting risk factors for high SeMspecific antibody titers after natural outbreaks of Streptococcus equi subsp equi infection in horses. J Am Vet Med Assoc 250, 1432-1439.

Boyle AG, Timoney JF, Newton JR, Hines MT, Waller AS, et al. 2018. Streptococcus equi infections in horses: guidelines for treatment, control, and prevention of strangles-revised consensus statement. $J$ Vet Intern Med 32, 633-647.

Chalker VJ, Waller A, Webb K, Spearing E, Crosse P, et al. 2012. Genetic diversity of Streptococcus equi subsp. zooepidemicus and doxycycline resistance in kennelled dogs. J Clin Microbiol 50, 2134-2136.

Chanter N, Newton J, Wood J, Verheyen K, Hannant D. 1998. Detection of strangles carriers. Vet Rec 142, 496.

Christmann U, Pink C. 2017. Lessons learned from a strangles outbreak on a large Standardbred farm. Equine Vet Educ 29, 138-143.

Dominguez M, Münstermann S, de Guindos I, Timoney P. 2016. Equine disease events resulting from international horse movements: Systematic review and lessons learned. Equine Vet J 48, 641-653.

Galan JE, Timoney JF. 1985. Mucosal nasopharyngeal immune responses of horses to protein antigens of Streptococcus equi. Infect Immun 47, 623-628.

Galan J, Timoney J. 1987. Molecular analysis of the M protein of Streptococcus equi and cloning and expression of the M protein gene in Escherichia coli. Infect Immun 55, 3181-3187.

Galan J, Timoney J. 1988. Immunologic and genetic comparison of Streptococcus equi isolates from the United States and Europe. $J$ Clin Microbiol 1142-1146.

Guss B, Flock M, Frykberg L, Waller AS, Robinson C, et al. 2009. Getting to grips with strangles: An effective multi-component recombinant vaccine for the protection of horses from Streptococcus equi infection. PLoS Pathog 5, e1000584.

Heath SE, Geor RJ, Tabel H, Mclntosh K. 1991. Unusual patterns of serum antibodies to Streptococcus equi in two horses with Purpura Hemorrhagica. J Vet Intern Med 5, 5263-267.

Hoffman A, Staempfli H, Prescott J, Viel L. 1991. Field evaluation of a commercial M-protein vaccine against Streptococcus equi infection in foals. Am J Vet Res 52, 589-592.

Ivens PAS, Matthews D, Webb K, Newton JR, Steward K, et al. 2011. Molecular characterisation of "strangles" outbreaks in the UK: The use of M-protein typing of Streptococcus equi ssp. equi. Equine Vet $\mathrm{J} 43,359-364$.
Jacobs A, Goovaerts D, Nuijten P, Theelen R, Hartford O, et al. 2000. Investigations towards an efficacious and safe strangles vaccine: submucosal vaccination with a live attenuated Streptococcus equi. Vet Rec 20, 563-567.

Judy CE, Chaffin MK, Cohen ND. 1999. Empyema of the guttural pouch (auditory tube diverticulum) in horses: 91 cases (1977-1997). J Am Vet Med Assoc 215, 1666-1670.

Kelly C, Bugg M, Robinson C, Mitchell Z, Davis-Poynter N, et al. 2006. Sequence variation of the SeM gene of Streptococcus equi allows discrimination of the source of strangles outbreaks. J Clin Microbiol 44, 480-486.

Kemp-Symonds J, Kemble T, Waller A. 2007. Modified live Streptococcus equi ('strangles') vaccination followed by clinically adverse reactions associated with bacterial replication. Equine Vet J 39, 284-286.

Knowles E, Mair T, Butcher N, Waller A, Wood J. 2010. Use of a novel serological test for exposure to Streptococcus equi subspecies equi in hospitalised horses. Vet Rec 166, 294-297.

Ling A, Upjohn M, Webb K, Waller A, Verheyen K. 2011. Seroprevalence of Streptococcus equi in working horses in Lesotho. Vet Rec 169, 72.

Mallicote M. 2015. Update on Streptococcus equi subsp equi infections. Vet Clin North Am - Equine Pract 31, 27-41.

Newton J, Wood J, Dunn K, DeBrauwere M, Chante N. 1997. Naturally occurring persistent and asymptomatic infection of the guttural pouches of horses with Streptococcus equi. Vet Rec 25, 84-90.

Parkinson NJ, Robin C, Newton JR, Slater J, Waller AS. 2011. Molecular epidemiology of strangles outbreaks in the UK during 2010. Vet $\operatorname{Rec} 168,666-701$.

Patty O, Cursons R. 2014. The molecular identification of Streptococcus equi subsp. equi strains isolated within New Zealand. NZ Vet J 2, 63-67.

Piche C. 1984. Clinical observations on an outbreak of strangles. Can Vet $J$ 25, 7-11.

Pusterla N, Kass PH, Mapes S, Johnson C, Barnett DC, et al. 2011. Surveillance programme for important equine infectious respiratory pathogens in the USA. Vet Rec 169, 12-18.

Pusterla N, Watson J, Affolter V, Magdesian K, Wilson W, et al. 2003. Purpura haemorrhagica in 53 horses. Vet Rec 4, 118-121.

Riihimaki M, Steward K, Pringle J. 2017. Persistence of Streptococcus equi ssp. equi Antibodies in a prolonged strangles outbreak, assessed by an enhanced indirect enzyme-linked immunosorbent assay. Equine Vet J 49, 23-24.

Robinson C, Steward KF, Potts N, Barker C, Hammond T, et al. 2013. Combining two serological assays optimises sensitivity and specificity for the identification of Streptococcus equi subsp. equi exposure. Vet J 197, 188-191.

Sheoran A, Sponseller B, Holmes M, Timoney J. 1997. Serum and mucosal antibody isotype responses to M-like protein (SeM) of Streptococcus equi in convalescent and vaccinated horses. Vet Immunol Immunopathol 59, 239-251.

Sheoran AS, Artiushin S, Timoney JF. 2002. Nasal mucosal immunogenicity for the horse of a SeM peptide of Streptococcus equi genetically coupled to cholera toxin. Vaccine 20, 1653-1659.

Sweeney C, Whitlock R, Meirs D, Whitehead S, Barningham S. 1987. Complications associated with Streptococcus equi infection on a horse farm. J Am Vet Med Assoc 191, 1446-1448.

Sweeney CR, Timoney JF, Newton JR, Hines MT. 2005. Streptococcus equi infections in horses: guidelines for treatment, control, and prevention of strangles. J Vet Intern Med 19, 123-134.

Timoney J, Kumar P. 2008. Early pathogenesis of equine Streptococcus equi infection (strangles). Equine Vet J 40, 637-642.

Timoney JF, Qin A, Muthupalani S, Artiushin S. 2007. Vaccine potential of novel surface exposed and secreted proteins of Streptococcus equi. Vaccine 25, 5583-5590.

Tiwari R, Qin A, Artiushin S, Timoney JF. 2007. Se18.9, an anti-phagocytic factor $\mathrm{H}$ binding protein of Streptococcus equi. Vet Microbiol $121,105-115$

Moreira R, García A, Ahumada C, Badía C, Suárez P, et al. 2019. Report of 2018 equine influenza outbreak in Chile, Austral J Vet Sci 51, 27-31. 
Verheyen K, Newton JR, Talbot NC, De Brauwere MN, Chanter N. 2000. Elimination of guttural pouch infection and inflammation in asymptomatic carriers of Streptococcus equi. Equine Vet J 32, 527-532.

Waller AS. 2014. New perspectives for the diagnosis, control, treatment, and prevention of strangles in horses. Vet Clin N Am - Equine 30, 591-607.
Webb K, Barker C, Harrison T, Heather Z, Steward KF, et al. 2013. Detection of Streptococcus equi subspecies equi using a triplex qPCR assay. Vet $J$ 195, 300-304.

Weese JS, Jarlot C, Morley PS. 2009. Survival of Streptococcus equi on surfaces in an outdoor environment. Can Vet J 50, 968-970. 
